\title{
Upper Airway Surgery for Obstructive Sleep Apnea Reduces Blood Pressure
}

\author{
Kenny P. Pang, FRCS(Ed), FRCSI(OTO) (D; Edward B. Pang; Kathleen A. Pang; Claudio Vicini, MD; \\ Yiong Huak Chan, BSc, PhD; Brian W. Rotenberg, MD, MPH, FRCSC (D)
}

\begin{abstract}
Objectives/Hypothesis: To evaluate if upper airway surgery reduces blood pressure in patients with obstructive sleep apnea (OSA).

Study Design: Prospective series.

Methods: A prospective series of 112 consecutive OSA patients with hypertension (HTN). All patients were $>18$ years old, respiratory disturbance index $>5$, all levels of apnea-hypopnea index (AHI), with a history of HTN treated with medication for at least 6 months. Surgical procedures included septoplasty, turbinate reduction, palate surgery, and tongue base reduction.
\end{abstract}

Results: There were 92 men and 20 women, with a mean age of 48.6 years, mean body mass index (BMI) was 27.5 (range, 19.7-34.7). Mean follow-up was 16.1 months. The mean preoperative AHI was 32.6 (range, 1.2-104), with the mean lowest oxygen saturation (LSAT) of 79.9\% (range, 52\%-93\%). The mean adjusted preoperative and postoperative systolic blood pressure (SBP) was reduced from $146 \pm 15.3 \mathrm{~mm} \mathrm{Hg}$ to $122 \pm 12.5 \mathrm{~mm} \mathrm{Hg}(P<.001)$, and diastolic blood pressure (DBP) was reduced from $91 \pm 10.2 \mathrm{~mm} \mathrm{Hg}$ to $76 \pm 7.8 \mathrm{~mm} \mathrm{Hg}(P<.001)$. There was a decrease in overall BMI from $27.5 \pm 3.6$ to $25.5 \pm 3.0(P<.001)$; however, based on multivariate analysis, the reduction in SBP and DBP was not affected by this BMI reduction. Fifty-eight patients (51.8\%) did not require their antihypertensive after surgery. There was poor correlation noted between HTN with AHI, LSAT, and oxygen duration $<90 \%$.

Conclusions: Upper airway surgery does reduce SBP and DBP in patients with OSA.

Key Words: Blood pressure, upper airway surgery, obstructive sleep apnea.

Level of Evidence: 4.

Laryngoscope, 00:000-000, 2017

\section{INTRODUCTION}

Obstructive sleep apnea (OSA) is a common sleep disorder. Young et al. studied 602 state employees with attended overnight polysomnography and found that the incidence of sleep-disordered breathing (SDB) was $24 \%$ in men and $9 \%$ in women. ${ }^{1}$ It is estimated that up to $93 \%$ of females and $82 \%$ of males with moderate to severe OSA remain undiagnosed. ${ }^{2}$ Obstructive sleep apnea is due to the collapsibility of the upper airway during sleep. These collapsible soft tissues, when subjected to negative pressure within the upper airway,

From the Department of Otorhinolaryngology Head and Neck Surgery (K.P.P.), Asia Sleep Center (E.B.P., K.A.P.), Singapore; G. B. MorgagniL. Pierantoni Hospital (c.v.), University of Pavia, Forli, Italy; Biostatistics Unit, Yong Loo Lin School of Medicine (Y.H.C.), Yong Loo Lin School of Medicine, National University, Singapore; Department of OtolaryngologyHead and Neck Surgery (B.w.R.), Western University, London, Ontario, Canada. $1,2017$.

Editor's Note: This Manuscript was accepted for publication June

K.P.P. conceived the study, collected the patients, performed the surgeries, and wrote the article. K.A.P., E.B.P., C.V., and B.w.R. wrote the results, conclusion, and methods. C.Y.H. performed the statistical analysis and wrote the statistical section.

The authors have no funding, financial relationships, or conflicts of interest to disclose.

Send correspondence to Kenny Peter Pang, Department of Otorhinolaryngology, Asia Sleep Centre, Paragon Medical Centre, Singapore 238859. E-mail: drkpang@gmail.com

DOI: 10.1002/lary.26759 may lead to complete or partial obstruction of the upper airway leading to cessation of breathing, increased sympathetic activity, increased blood pressure (BP), and hypoxemia. Multilevel upper airway surgery (similar to continuous positive airway pressure [CPAP] therapy) has been shown to be more efficacious in this recent decade, not only in terms of improving quality of life but also reducing the apnea-hypopnea index [AHI]. ${ }^{3-6}$

The Sleep Heart Health Study and the Wisconsin Sleep Cohort have demonstrated a strong link between OSA and hypertension. ${ }^{78}$ This is believed to be due to sleep fragmentation, nocturnal hypoxemia, and increased sympathetic tone. ${ }^{9}$ This increased sympathetic tone is manifested not only during the nocturnal hypoxic events but also during the day as systemic hypertension. The physiologic changes that are the result of recurrent apneas and hypoxemia can cause acute thrombotic events, atherosclerosis, and cerebrovascular accidents. There is convincing evidence of the association between OSA and cardiovascular (CV) disease. ${ }^{8}$ There is a higher mortality rate among patients with CV disease who also have OSA. ${ }^{7-9}$

When patients present for treatment of OSA, the main goal of therapy should not be to reduce AHI, but rather to improve target outcome measures that are either subjective (such as patient wakefulness or quality of life), and improve end-organ damage (most commonly considered to be hypertension [HTN]). To date, very few studies in the surgical literature have focused on $\mathrm{BP}$ as 
TABLE I.

Showing the Respective Decrease in Systolic and Diastolic Blood Pressure, Preoperatively and Postoperatively, Based on AHI Severity

\begin{tabular}{|c|c|c|c|c|c|c|}
\hline \multirow[b]{2}{*}{$\mathrm{AHI}$} & \multirow[b]{2}{*}{ No. of Patients } & \multicolumn{2}{|c|}{$\mathrm{SBP}, \mathrm{mm} \mathrm{Hg}$} & \multicolumn{2}{|c|}{ DBP, $\mathrm{mm} \mathrm{Hg}$} & \multirow[b]{2}{*}{$P$ Value } \\
\hline & & Preoperatively & Postoperatively & Preoperatively & Postoperatively & \\
\hline $5-15$ & 26 & 141 & 121 & 85.6 & 75.3 & $<.001$ \\
\hline $15-30$ & 28 & 143 & 123 & 89.8 & 75.9 & $<.001$ \\
\hline$>30$ & 54 & 150 & 122 & 91.8 & 76.2 & $<.001$ \\
\hline
\end{tabular}

$\mathrm{AHI}=$ apnea-hypopnea index; DBP = diastolic blood pressure; SBP = systolic blood pressure.

the main outcome measure, but this is a delinquency necessitating correction. We present our study on 112 patients with SDB who have reduced their systolic blood pressure (SBP) and diastolic blood pressure (DBP) after upper airway surgery.

\section{MATERIALS AND METHODS}

This was a nonrandomized prospective clinical study of 112 consecutively collected patients with OSA and HTN. Patients were evaluated in the snoring/sleep subspecialty clinic. The inclusion criteria were age $>18$ years, all body mass index numbers (BMIs), all tonsil size grades, all Friedman clinical stages, retropalatal and/or retroglossal obstruction, no previous oronasal surgical procedures, and all AHIs. All included patients were diagnosed with hypertension $>6$ months prior to surgery date and were on some form of antihypertensive medications. The study protocol and methodology was reviewed and approved by the hospital ethics committee/institutional review board. All procedures performed in studies involving human participants were in accordance with the ethical standards of the institutional and/or national research committee and with the 1964 Helsinki declaration and its later amendments or comparable ethical standards.

All patients underwent a comprehensive clinical assessment including a thorough physical examination, blood pressure readings, nasoendoscopy, and an overnight polysomnography. Patients completed the Epworth Sleepiness Scale (ESS) and a visual analogue scale (VAS) for snoring before and after surgery. The sleep partner completed a similar scale for snoring. The patient also completed a VAS for pain on postoperative days 1 , 3,7 , and 14. Examination included height, weight, neck circumference, BMI, and blood pressure, and an endoscopic assessment of the nasal cavity, posterior nasal space, oropharyngeal area, soft palatal redundancy, uvula size and thickness, tonsillar size, and Friedman grade. Patients who complained of nasal obstruction and/or persistent runny nose with clinical endoscopic findings of either turbinate hypertrophy, septal deviation, and/or nasal polyps were offered nasal surgery at the same sitting as the palate/tongue surgery. The nasal surgery included endoscopic sinus surgery, septoplasty, and/or bilateral radiofrequency turbinate reduction. Patients with retropalatal collapse underwent palatal surgery (either expansion sphincter pharyngoplasty, anterior palatoplasty, uvulopalatopharyngoplasty with or without tonsillectomy/tonsillotomy) based on upper airway evaluation. Tongue surgery included tongue base radiofrequency volumetric reduction. All patients were given dietary advice regarding daily calorie counts and intake. There were no dropouts or withdrawals in this study.

The primary outcome measure of this study was change in SBP or DBP, as well as notation regarding the need for ongoing BP medications after surgery. Secondary outcomes were changes in BMI, subjective improvement in snoring based on the VAS, and improvement in sleepiness as indicated by the ESS.

\section{Statistical Analysis}

All analyses were performed using SPSS 24.0 (IBM, Armonk, NY) with statistical significance set at $P<.05$. Descriptive for numerical variables were presented as mean (range) and number $(\%)$ for categorical variables. Pre-post comparisons for SBP, DBP, ESS scores, and snore scores were assessed using paired $t$ test and adjusted for BMI and demographical variables using mixed model. Overall subjects and subgroup analyses by AHI levels $(<5,5$ to $<15,15$ to $<30, \geq 30)$ were performed. Pearson correlation was presented for SBP and DBP with AHI, and lowest oxygen saturation and oxygen duration below $90 \%$.

\section{RESULTS}

There were a total of 92 men and 20 women; 93 were Chinese, eight were Caucasian, six were Indian, and five were Malay. Their mean age was 48.6 years old (range, 28-67 years), mean BMI was 27.5 (range, 19.734.7 , with a mean follow-up of 16.1 months. The mean preoperative respiratory disturbance index was 40 (range, 11-104), AHI was 32.6 (range, 1.2-104), with the mean lowest oxygen saturation (LSAT) of $79.9 \%$ (range, $52 \%$ to $93 \%$ ). The mean preoperative and postoperative SBP (adjusted and controlled for BMI, age, race, and gender) was reduced from $146 \pm 15.3 \mathrm{~mm} \mathrm{Hg}$ to $122 \pm$ $12.5 \mathrm{~mm} \mathrm{Hg}(P<.001)$; the percentage change was a $16.4 \%$ decrease in systolic pressure. The mean preoperative and postoperative DBP (adjusted for BMI, age, race, and gender) was reduced from $91 \pm 10.2 \mathrm{~m} \mathrm{mHg}$ to $76 \pm$ $7.8 \mathrm{~mm} \mathrm{Hg}(P<.001)$; the percentage change was a $16.5 \%$ decrease in diastolic pressure. ESS score improved from $11.54 \pm 4.1$ to $4.88 \pm 1.4(P<.001)$, and the VAS snore score improved from $7.7 \pm 0.8$ to $2.3 \pm 0.9(P<.001)$. There was a decrease in overall BMI from $27.5 \pm 3.6$ to $25.5 \pm 3.0(P<.001)$; however, based on multivariate analysis, the reduction in BP was not affected by this BMI reduction.

We divided the cohort based on severity of OSA. There were 4 patients who had a normal AHI, but wanted the nose and palate surgery as they had extremely loud snoring that bothered their bed partners. Their SBP and DBP (adjusted for BMI, age, race, and gender) also decreased postoperatively from $148 \pm$ $11.5 \mathrm{~mm} \mathrm{Hg}$ to $127 \pm 11.4 \mathrm{~mm} \mathrm{Hg}$ and $86.9 \pm 2.1 \mathrm{~mm} \mathrm{Hg}$ to $65.9 \pm 1.2 \mathrm{~mm} \mathrm{Hg}$, respectively (not statistically significant due to the small sample size in this group). There were 26 patients in the mild OSA group (AHI 5-15), and 
TABLE II.

Showing the Respective Change in Systolic and Diastolic Blood Pressure, Preoperatively and Postoperatively, Based on AHI Severity

\begin{tabular}{lcccrr}
\hline AHI & No. of Patients & Decrease in SBP, mm Hg & $\%$ & Decrease in DBP, mm Hg & $\%$ \\
\hline $5-15$ & 26 & 20.0 & $14.1 \%$ & 10.3 & $11.7 \%$ \\
$15-30$ & 28 & 20.0 & $13.9 \%$ & 13.9 & $15.5 \%$ \\
$>30$ & 54 & 27.0 & $18.0 \%$ & 15.6 & $<.001$ \\
\hline \hline
\end{tabular}

$\mathrm{AHI}=$ apnea hypopnea index; $\mathrm{DBP}=$ diastolic blood pressure; $\mathrm{SBP}=$ systolic blood pressure.

their SBP and DBP (adjusted for BMI, age, race, and gender) also decreased postoperatively from $141 \pm$ $25.6 \mathrm{~mm} \mathrm{Hg}$ to $121 \pm 23.6 \mathrm{~mm} \mathrm{Hg}$ (a $14.1 \%$ decrease in systolic pressure) and $85.6 \pm 10.1 \mathrm{~mm} \mathrm{Hg}$ to $75.3 \pm$ $8.8 \mathrm{~mm} \mathrm{Hg}$ (an $11.7 \%$ decrease in diastolic pressure), respectively $(P<.001)$ (Table I). There were 28 patients who had moderate OSA (AHI 15-30), and their SBP and DBP (adjusted for BMI, age, race, and gender) decreased postoperatively from $143 \pm 19.1 \mathrm{~mm} \mathrm{Hg}$ to $123 \pm 12.7 \mathrm{~mm}$ $\mathrm{Hg}$ (a $13.9 \%$ decrease in systolic pressure) and $89.8 \pm$ $12.6 \mathrm{~mm} \mathrm{Hg}$ to $75.9 \pm 6.6 \mathrm{~mm} \mathrm{Hg}$ (a $15.5 \%$ decrease in diastolic pressure), respectively $(P<.001)$ (Table I). Severe OSA $(\mathrm{AHI}>30)$ was the largest group, with a total of 54 patients, and postoperatively their SBP and DBP (adjusted for BMI, age, race, and gender) encouragingly decreased postoperatively from $150 \pm 12.9 \mathrm{~mm} \mathrm{Hg}$ to $122 \pm 12.3 \mathrm{~mm} \mathrm{Hg}$ (an 18\% decrease in systolic pressure) and $91.8 \pm 9.2 \mathrm{~mm} \mathrm{Hg}$ to $76.2 \pm 7.8 \mathrm{~mm} \mathrm{Hg}$ (a $17.1 \%$ decrease in diastolic pressure), respectively $(P<.001)$ (Tables I and II). There was a documented decrease in BMI in each group; however, this decrease in BMI did not statistically affect the outcome.

Correlation of SBP and DBP with AHI was poor at $r=0.11$ and $r=0.18$, respectively. Similarly, the correlation of SBP and DBP with LSAT was $r=0.05$ and $r=0.09$; however, there was mild correlation between DBP and oxygen duration below $90 \%$ with $r=0.2$, whereas $r=0.06$ was the correlation between SBP and oxygen duration below $90 \%$.

The overall ESS score improved from $11.54 \pm 4.1$ to $4.88 \pm 1.4(P<.001)$, and the VAS snore score improved from $7.7 \pm 0.8$ to $2.3 \pm 0.9(P<.001)$. Of note, 58 out of 112 patients $(51.8 \%)$ did not require their antihypertensive medications anymore and were normotensive. An additional 31 patients $(27.7 \%)$ were on half their initial preoperative dosage and/or reduced their preoperative antihypertensive dosage. All patients were generally pleased with their postoperative outcome.

\section{DISCUSSION}

In a typical normal person, at night, the $\mathrm{BP}$ would have an average of $10 \%$ to $20 \%$ dip/drop in their SBP compared to their BP during the day. It is believed that in patients with mild or developing OSA, during the night, the BP might not drop at all; these patients are called "nondippers." In patients with moderate or severe OSA, BP surges can be observed in both systemic and pulmonary circulation ${ }^{10}$; this can be attributed to the sympathetic activity. Similarly, cyclical variations of the heart rate may also be evident (i.e., sinus tachycardia/ bradycardia) in these OSA patients. ${ }^{11}$ Furthermore, it has been documented that arrhythmias may occur in patients with severe OSA during the night during a profound hypoxemic event. ${ }^{12}$ These include sinus arrests, atrioventricular conduction blocks, atrial fibrillation, and ventricular arrhythmias. It is also believed that through these arrhythmias, OSA may even cause sudden cardiac death; however, the evidence for this is still somewhat circumstantial. ${ }^{13}$

The detrimental effects of OSA on the CV system are carried over into daytime hours. It is known that up to one-half of these patients suffer from arterial hypertension. The OSA-associated arterial hypertension is characterized not only by nocturnal hypertension, but a high percentage of these OSA patients have refractory and masked hypertension, requiring dual or multipharmacotherapy. ${ }^{14-16}$ Mainly through its pressor effects, OSA increases the risks for stroke, heart failure, and myocardial infarction. ${ }^{17} \mathrm{CV}$ risk in OSA depends on the severity of their OSA; that is, those patients with an AHI exceeding 30 per hour (severe OSA) of sleep are affected to a larger extent. ${ }^{17}$ It is well accepted that patients with OSA have a higher incidence of hypertension, and some studies have showed as high as a 1.5 to 2.7 times higher incidence of hypertension associated with OSA. ${ }^{18-20}$ Moreover, the incidence of OSA is very common in hypertensive patients, and the prevalence has been reported to be as high as $56 \% .^{21,22}$

In the medical literature, treatment of OSA patients with CPAP have consistently and reliably showed a decrease in BP. The mechanisms involved in this beneficial effect of CPAP on the vascular system likely include a reduction of $\mathrm{BP}$ and improvement of vascular function. ${ }^{23-26}$ A randomized controlled trial (RCT) conducted by Weaver et al. ${ }^{27}$ evaluated the efficacy of CPAP treatment on functional status assessed by the Functional Outcomes of Sleep Questionnaire in sleepy patients with mild and moderate OSA. They assessed a total of 239 patients with a mean AHI of about 13/hour; they were randomized to CPAP treatment or placebo. After 8 weeks, CPAP treatment significantly improved the functional outcome of patients with mild OSA, and there was also a significant change in daytime DBP values from baseline by $-1.93 \mathrm{~mm} \mathrm{Hg}$ (95\% confidence interval $[\mathrm{CI}]:-3.8$ to $0.0 ; P=.048$ ) between the two groups. ${ }^{27}$ In the Multicentre Obstructive Sleep Apnoea Interventional Cardiovascular (MOSAIC) trial of 391 patients with OSA, they were randomized to 6 months of autoadjusting CPAP therapy or standard care. The authors demonstrated that CPAP treatment significantly 
improved subjective daytime sleepiness (adjusted treatment effect on ESS $-2.0 ; 95 \%$ CI: -2.6 to -1.4 ; $P<.0001)$. However, this positive treatment effect on symptoms was not accompanied by a reduction in $\mathrm{BP}^{28}$ The findings of the MOSAIC study were confirmed by a meta-analysis published by Bratton et al., ${ }^{29}$ in which the individual data of 1,206 patients from four RCTs were evaluated. Although CPAP treatment reduced OSA severity and sleepiness in minimally symptomatic patients, CPAP did not to have a beneficial effect on BP, except in those patients who used CPAP for $>4$ hours/ night, suggesting that a minimum of 4 hours use per night is needed. ${ }^{29}$

There are a number of published RCTs on the effect of CPAP on BP in patients with resistant hypertension. ${ }^{30,31}$ Lozano et al. ${ }^{30}$ had 64 patients randomized to receive CPAP versus conventional medical treatment alone. After a 3 month period, patients who used CPAP $>5.8$ hours showed a greater reduction than patients treated with standard medication in daytime DBP ( $-6.12 \mathrm{~mm} \mathrm{Hg} ; 95 \% \mathrm{CI}:-1.45$ to $-10.82 ; P=.004)$, 24hour DBP $(6.98 \mathrm{~mm} \mathrm{Hg} ; 95 \% \mathrm{CI}$ : -1.86 to -12.1 ; $P=.009)$, and 24-hour SBP $(-9.71 \mathrm{~mm} \mathrm{Hg}$; 95\% CI: -0.20 to $-19.22 ; P=.046)$. Moreover, the number of patients with the nocturnal dipping pattern significantly increased in the CPAP group compared to conventional medical treatment ( $51.7 \%$ vs. $24.1 \%, P=.008){ }^{31}$

In 2014, Schein et al. ${ }^{32}$ reviewed 16 RCTs that included 1,166 OSA patients in total, and demonstrated that CPAP usage resulted in clinically relevant reductions of BP. CPAP treatment was associated with a reduction of SBP by $3.20 \mathrm{~mm} \mathrm{Hg}$ (95\% CI: 1.72 to 4.67 ) and $\mathrm{DBP}$ by $2.87 \mathrm{~mm} \mathrm{Hg}$ (95\% CI: 0.55 to 5.18$){ }^{32} \mathrm{~A}$ meta-analysis by Montesi et al. ${ }^{33}$ included 32 RCTs that showed similar results, with OSA patients treated with CPAP benefitting from significant reductions in SBP by $2.58 \mathrm{~mm} \mathrm{Hg}$ (95\% CI: 3.57 to 1.59$)$ and DBP by $2.01 \mathrm{~mm}$ $\mathrm{Hg}$ (95\% CI: 2.84 to 1.18). Nighttime SBP had the most prominent reduction after treatment with CPAP (4.09 mm Hg; 95\% CI: 6.24 to 1.94$).{ }^{33}$ In another recently published meta-analysis of 29 RCTs that included 1,820 patients, Fava et al. also showed a decreased SBP $(2.6 \pm$ $0.6 \mathrm{~mm} \mathrm{Hg})$ and DBP $(2.0 \pm 0.4 \mathrm{mmHg})$ in patients with CPAP treatment. ${ }^{34}$

It is well accepted, as illustrated above ${ }^{28-34}$ that CPAP usage does reduce BP; the caveat is that compliance is the issue and the patient has to use it throughout the night. Although there are not many published data on the effect of upper airway surgery in OSA patients on BP, most published data do show some marginal decrease in BP following surgery. ${ }^{35-38}$ De Paula Seares et al. showed in a small 18-patient group with OSA, that following upper airway surgery, the mean systolic pressure reduced by $7.4 \mathrm{~mm} \mathrm{Hg}$, whereas the mean diastolic pressure reduced by $4.2 \mathrm{~mm} \mathrm{Hg}$ at a 6 -month follow-up. $^{36}$ Lee et al. demonstrated a decrease of between 6 and $10 \mathrm{~mm} \mathrm{Hg}$ in 50 children with OSA, after adenotonsillectomy. ${ }^{37}$ Similarly, in 78 children with OSA, Kuo et al. showed a decrease in systolic pressure of between 5.4 to $10.9 \mathrm{~mm} \mathrm{Hg}$ and diastolic pressure between 12.0 and $18.8 \mathrm{~mm} \mathrm{Hg}{ }^{38}$
TABLE III.

Showing Cohen's Effect of Body Mass Index Change on Preoperative and Postoperative Respective Parameters

\begin{tabular}{lc}
\hline & Cohen's $d$ \\
\hline SBP & 0.05 \\
DBP & 0.14 \\
Epworth & 0.08 \\
Snore & 0.21 \\
\hline \hline
\end{tabular}

Cohen's interpretation: greater than 0.8, large effect; between 0.50.8 , moderate effect;

less than 0.5 , small effect.

$\mathrm{SBP}=$ systolic blood pressure; $\mathrm{DBP}=$ diastolic blood pressure

In our series, we demonstrated that with strict dietary control, weight loss, and upper airway surgery in tandem, we managed to achieve a clinically significant decrease in SBP (16.4\%) and DBP (18\%) following surgery. The greatest decrease in $\mathrm{BP}$ was noted in the group of patients $(\mathrm{n}=54)$ with severe OSA (AHI > 30); there was an $18 \%$ decrease (mean $=27 \mathrm{~mm} \mathrm{Hg}$ ) in SBP and $17.1 \%$ decrease $($ mean $=15.6 \mathrm{~mm} \mathrm{Hg})$ in DSP. We attributed this specific group (severe OSA) to have the largest decrease in BP as they started out (preoperatively) with the highest mean BP. Half of the entire group of 112 patients $(51.8 \%)$ did not require their antihypertensive medications postoperatively, and 31 patients $(27.7 \%)$ had their initial preoperative dosage reduced.

After statistically adjusting for BMI, age, race, and gender, the decrease in both SBP and DBP was significant in all three groups. We acknowledge that there was an overall decrease in BMI from $27.5 \pm 3.6$ to $25.5 \pm 3.0$ $(P<.001)$, which could have had an impact on the overall decrease of the postoperative BP (although based on the multivariate analysis, the reduction in $\mathrm{BP}$ was not affected by this BMI reduction). It is pertinent to note that the outcome of interest is the change in $\mathrm{BP}$, and assessing the variables (change in BMI, age, race, and gender) that affect the outcome of interest (BP), none of the variables were significant, and hence, the change in BMI had no influence in the change in BP. Based on Cohen's effect of BMI change on the preoperative and postoperative BP readings, ESS, and Snore VAS (Table III), it is evident that the change in BMI had little or no effect in the changes noted for these parameters.

The overall changes in these OSA patients' inner milieu have a significant impact in reducing the patient's oxidative stress from the systemic effects of OSA; the drop in BP and reduction in BMI are important positive changes in the OSA patient's systemic health. As sleep physicians treating patients with OSA, perhaps we should consider other more pertinent systemic parameters that affect the patient's overall oxidative stress, rather than one single parameter called the AHI (which is nebulous to the patient), and consider systemic parameters like BP and BMI. ${ }^{39,40}$

We acknowledge the limitations of this study, in that the patient numbers were not large, most patients were Asian, follow-up was 16 months, and not all patients had a postoperative sleep test done. 


\section{CONCLUSION}

Overall, this is an important issue in the outcome of treatment for the patient with OSA; not often addressed, but crucial as it affects the patient as a whole, including the oxidative stress that takes place in a patient suffering from repetitive episodes of hypoxia every night. Our data in 112 patients with OSA demonstrate that upper airway surgery can be effective in reducing postoperative SBP and DBP and provide the hope of eliminating the need for oral antihypertensive therapy.

\section{BIBLIOGRAPHY}

1. Young T, Palta M, Dempsey J, et al. The occurrence of SDB among middle-aged adults. $N$ Engl J Med 1993;328:1230-1235.

2. Young T, Evans L, Finn L, et al. Estimation of the clinically diagnosed proportion of sleep apnea syndrome in middle aged men and women. Sleep 1997;20:705-706

3. Baldwin CM, Griffith KA, Nieto FJ, O'Connor GT, Walsleben JA, Redline $\mathrm{S}$. The association of sleep-disordered breathing and sleep symptoms with quality of life in the Sleep Heart Health Study. Sleep 2001;24:96105

4. D'Ambrosio C, Bowman T, Mohsenin V. Quality of life in patients with obstructive sleep apnea: effect of nasal continuous positive airway pressure--a prospective study. Chest 1999;115:123-129.

5. Jenkinson C, Stradling J, Petersen S. Comparison of three measures of quality of life outcome in the evaluation of continuous positive airways pressure therapy for sleep apnoea. J Sleep Res 1997;6:199-204.

6. Woodson BT, Steward DL, Weaver EM, Javaheri S. A randomized trial of temperature-controlled radiofrequency, continuous positive airway pressure, and placebo for obstructive sleep apnea syndrome. Otolaryngol Head Neck Surg 2003;128:848-861.

7. Nieto JF, Young TB, Lind BK, Shahar E. Association of SDB, sleep apnea and hypertension in a large community based study. JAMA 2000;283: 1829-1836.

8. Peppard PE, Young T, Palta M, Skatrund J. Prospective study of the association between SDB and hypertension. N Engl J Med 2000;342:13781384

9. Somers VK, Dyken ME, Clary MP, Abbond FM. Sympathetic neural in OSA. J Clin Invest 1995;96:1897-1904.

10. Marrone O, Bonsignore MR. Pulmonary haemodynamics in obstructive sleep apnoea. Sleep Med Rev 2002;6:175-193.

11. Guilleminault $\mathrm{C}$, Connolly $\mathrm{S}$, Winkle $\mathrm{R}$. Cyclical variation of the heart rate in sleep apnoea syndrome. Mechanisms, and usefulness of $24 \mathrm{~h}$ electrocardiography as a screening technique. Lancet 1984;1:126-131.

12. Rossi VA, Stradling JR, Kohler M. Effects of obstructive sleep apnoea on heart rhythm. Eur Respir $J$ 2013:41:1439-1451.

13. Gami AS, Olson EJ, Shen WK, et al. Obstructive sleep apnea and the risk of sudden cardiac death: a longitudinal study of 10,701 adults. J Am Coll Cardiol 2013;62:610-616.

14. Wolf J, Hering D, Narkiewicz K. Non-dipping pattern of hypertension and obstructive sleep apnea syndrome. Hypertens Res 2010;33:867-871.

15. Logan AG, Perlikowski SM, Mente A, et al. High prevalence of unrecognized sleep apnoea in drug-resistant hypertension. J Hypertens 2001;19 2271-2277.

16. Baguet J-P, Lévy P, Barone-Rochette G, et al. Masked hypertension in obstructive sleep apnea syndrome. J Hypertens 2008;26:885-892.

17. Marin JM, Carrizo SJ, Vicente E, Agusti AGN. Long-term cardiovascular outcomes in men with obstructive sleep apnoea-hypopnoea with or without treatment with continuous positive airway pressure: an observational study. Lancet 2005;365(9464):1046-1053.

18. Calhoun DA, Jones D, Textor S, et al. Resistant hypertension: diagnosis, evaluation, and treatment: a scientific statement from the American Heart Association Professional Education Committee of the Council for High Blood Pressure Research. Circulation 2008;117:e510-e526.

19. Muiesan ML, Salvetti M, Rizzoni D, et al. Resistant hypertension and target organ damage. Hypertens Res 2013;36:485-491.
20. Pedrosa RP, Drager LF, Gonzaga CC, et al. Obstructive sleep apnea: the most common secondary cause of hypertension associated with resistant hypertension. Hypertension 2011;58:811-817.

21. Drager LF, Genta PR, Pedrosa RP, et al. Characteristics and predictors of obstructive sleep apnea in patients with systemic hypertension. Am J Cardiol 2010;105:1135-1139.

22. Calhoun DA, Harding SM. Sleep and hypertension. Chest 2010;138:434443.

23. Parati G, Lombardi C, Hedner J, et al. Position paper on the management of patients with obstructive sleep apnea and hypertension: joint recommendations by the European Society of Hypertension, by the European Respiratory Society and by the members of European COST (COoperation in Scientific and Technological research) ACTION B26 on obstructive sleep apnea. J Hypertens 2012;30:633-646.

24. Somers VK, White DP, Amin R, et al. Sleep apnea and cardiovascular disease: an American Heart Association/american College Of Cardiology Foundation Scientific Statement from the American Heart Association Council for High Blood Pressure Research Professional Education Committee, Council on Clinical Cardiology, Stroke Council, and Council On Cardiovascular Nursing. In collaboration with the National Heart, Lung, and Blood Institute National Center on Sleep Disorders Research (National Institutes of Health). Circulation 2008;118:1080-1111.

25. Milleron $\mathrm{O}$, Pillière $\mathrm{R}$, Foucher $\mathrm{A}$, et al. Benefits of obstructive sleep apnoea treatment in coronary artery disease: a long-term follow-up study. Eur Heart $J$ 2004;25:728-734.

26. Marin JM, Carrizo SJ, Vicente E, et al. Long-term cardiovascular outcomes in men with obstructive sleep apnoea-hypopnoea with or without treatment with continuous positive airway pressure: an observational study. Lancet 2005;365:1046-1053.

27. Weaver TE, Mancini C, Maislin G, et al. Continuous positive airway pressure treatment of sleepy patients with milder obstructive sleep apnea: results of the CPAP Apnea Trial North American Program (CATNAP) randomized clinical trial. Am J Respir Crit Care Med 2012;186:677-683.

28. Craig SE, Kohler M, Nicoll D, et al. Continuous positive airway pressure improves sleepiness but not calculated vascular risk in patients with minimally symptomatic obstructive sleep apnoea: the MOSAIC randomised controlled trial. Thorax 2012;67:1090-1096.

29. Bratton DJ, Stradling JR, Barbé $\mathrm{F}$, et al. Effect of CPAP on blood pressure in patients with minimally symptomatic obstructive sleep apnoea: a meta-analysis using individual patient data from four randomised controlled trials. Thorax 2014;69:1128-1135.

30. Pedrosa RP, Drager LF, de Paula LK, et al. Effects of OSA treatment on $\mathrm{BP}$ in patients with resistant hypertension: a randomized trial. Chest 2013:144:1487-1494.

31. Lozano L, Tovar JL, Sampol G, et al. Continuous positive airway pressure treatment in sleep apnea patients with resistant hypertension: a randomized, controlled trial. J Hypertens 2010;28:2161-2168.

32. Schein AS, Kerkhoff AC, Coronel CC et al Continuous positive airway pressure reduces blood pressure in patients with obstructive sleep apnea; a systematic review and meta-analysis with 1000 patients. J Hypertens 2014;32:1762-1773.

33. Montesi SB, Edwards BA, Malhotra A, et al. The effect of continuous positive airway pressure treatment on blood pressure: a systematic review and meta-analysis of randomized controlled trials. J Clin Sleep Med 2012;8:587-596.

34. Fava C, Dorigoni S, Dalle Vedove F, et al. Effect of CPAP on blood pressure in patients with OSA/hypopnea a systematic review and meta-analysis. Chest 2014;145:762-771.

35. Rotenberg BW, Theriault J, Gottesman S. Redefining timing for surgery in OSA in anatomically favourable patients. Laryngoscope 2014;124(suppl 4):S1-S9.

36. De Paula Seares CF, Cavichio L. Cahali MB. Lateral Pharyngoplasty reduces nocturnal blood pressure in patients with OSA. Laryngoscope 2014;124:311-316

37. Lee LA, Li HY, Lim YS, et al. Severity of childhood OSA and hypertension improved after adenotonsillectomy. Otolaryngol Head and Neck Surg 2015;152:553-560.

38. Kuo YL, Kang KT, Chiu SN et al. Blood pressure after surgery among obese and nonobese children with OSA. Otolarygol Head and Neck Surg 2015;152:931-940.

39. Pang KP, Rotenberg BW. Redefining successful therapy in obstructive sleep apnea: a call to arms. Laryngoscope 2014;124:1051-1052.

40. Pang KP, Rotenberg BW. The SLEEP GOAL as a success criteria in OSA therapy. Eur Arch Otorhinolaryngol 2016;273:1063-1065. 